\title{
Antimicrobial coatings - obtaining and characterization
}

\author{
CORNELIA GURAN, ALEXANDRA PICA ${ }^{\dagger}$, DENISA FICAI* ${ }^{*}$ ANTON FICAI \\ and CEZAR COMANESCU \\ Politehnica University of Bucharest, 1-7 Polizu St, Bucharest 011061, Romania \\ ${ }^{\dagger}$ Research Institute for Advanced Coating, 49 A Theodor Pallady Bd, Bucharest 032258, Romania
}

MS received 27 February 2012; revised 7 May 2012

\begin{abstract}
In this paper, we present inorganic-organic hybrid coatings with polymer matrix (water soluble) that contain silver nanoparticles (AgNPs). The structure and morphology of coating materials were determined by infrared spectroscopy (FT-IR) and scanning electron microscopy (SEM). Therefore, the antimicrobial activities and mechanisms of coatings for several pathogenic bacteria (Bacilius cereus and Staphylococcus aureus) were investigated. It was demonstrated that the obtained material with silver nanoparticles keep their antimicrobial effect even if they are subjected to several cycles of washing with water and detergent.
\end{abstract}

Keywords. Silver nanoparticles; antimicrobial coatings; antimicrobial activity.

\section{Introduction}

With the advent of nanotechnology, it was attempted to replace the biocides from antimicrobial paints with various nano-sized substances such as: zinc oxide, titanium dioxide and silver (Niegisch et al 2002). The development of antimicrobial nanocoatings through the green chemistry methods could be a promising way for potential environmental applications (Yamauchi et al 2005). These materials can be obtained either by adding nanopowders that are usually in organic-polymeric suspensions in the coating material or by in situ synthesis of nanopowders in the process of obtaining the coating material (Ross 2004).

The interest for antimicrobial coating materials based on silver is because of the low toxicity of silver on human cells, long biocide action, high thermal ability and low volatility (Thölmann et al 2003). Silver has a broad spectrum of biocide activity against 650 bacteria, mushrooms and viruses (Barmatov et al 2008). Silver in neutral state (as silver nanoparticles) has also antimicrobial properties (Sauvet et al 2003). Although the mechanism is not fully understood, it was shown that the AgNP interacts with the outer membrane of bacteria, causing structural changes and eventually causing its death (Borkow 2005). The antimicrobial action of the coating material is explained by the continuous release of silver in the material throughout its life (Hung and Hsu 2007).

The aim of this study was to improve the antimicrobial activity of the styrene acrylate-based coating material by the addition of various amounts of silver nanoparticles (200-650 ppm) with an average size of 40-60 nm.

\footnotetext{
*Author for correspondence (manzu_denisa76@yahoo.com)
}

\section{Experimental}

\subsection{Materials}

All the used chemicals were of analytical grade. Dispersion agents (pigment disperser: A and S) were supplied by BASF, while the used surfactant (dioctyl sodium sulfosuccinate) was supplied by Sigma-Aldrich. Acrylic-styrene polymer dispersion with free carboxyl groups was used as film-forming agent, the minimum film-forming temperature was $20^{\circ} \mathrm{C}$. Calcium carbonate and hydrated magnesium silicate were used as extenders while titanium dioxide (rutile) was used as white pigment. For antiseptic reasons, 200 $650 \mathrm{ppm}$ silver nanoparticles with particle size of 40-60 nm were used. The preparation and characterization of these nanoparticles were presented in a previous paper (Pica et al 2012).

\subsection{Equipment}

Vibrational spectra were recorded using a Shimadzu 8400 spectrometer in the wavenumbers range of $400-4000 \mathrm{~cm}^{-1}$.

UV-vis spectra were recorded on a Thermo Evolution 300 spectrometer, in absorbance mode, between 250 and $740 \mathrm{~nm}$.

SEM analysis was performed on a HITACHI S2600N electron microscope, at 25 and $15 \mathrm{keV}$, in primary electrons fascicle, on samples covered with a thin silver layer. For quantitative analyses uncovered samples were used.

\subsection{Obtaining of AgNPs-based antimicrobial coatings}

The most important factors in obtaining antimicrobial coating materials are: colloidal stability of the coatings; adhesion of coatings and efficient dispersion of nano-AgNP in the polymer matrix. 
Table 1. Conditions of preparation of antimicrobial film-forming materials.

\begin{tabular}{lcc}
\hline $\begin{array}{l}\text { Film-forming } \\
\text { materials }\end{array}$ & $\begin{array}{c}\text { Dispersion } \\
\text { time (h) }\end{array}$ & $\begin{array}{c}\text { AgNP content } \\
(\mathrm{ppm})\end{array}$ \\
\hline AM1 & 2 & 650 \\
AM2 & 1 h 30 min & 400 \\
AM3 & 2 & 350 \\
AM4 & 1 & 300 \\
AM5 & 1 & 200 \\
\hline
\end{tabular}

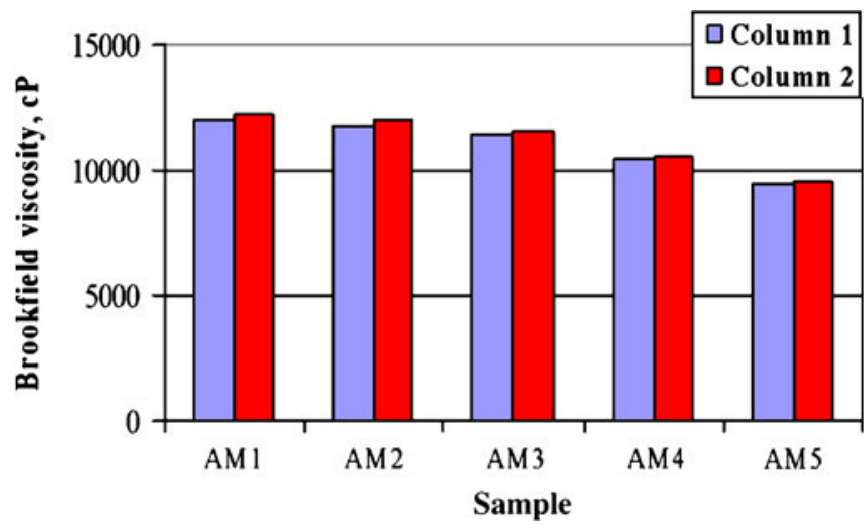

Figure 1. Shelf stability at 30 days under ambient conditions (column 1 - blue) and accelerated aging conditions at $50{ }^{\circ} \mathrm{C}$ (column 2 -red).
The colloidal stability during production of a film-forming material has a significant effect on the final distribution of particles in the film-forming material and therefore on its properties. The basic components of the antibacterial coating materials are: Ag nanoparticles, acrylic resin, pigments, fillers, extenders and additives. Both the type and the relative amount of these components can vary, resulting in a large number of different compositions of film-forming material. For this reason and because of the interactions that occur with other film-forming constituents, AgNPs were added to a conventional film-forming material.

\subsection{Preparation of conventional film-forming material}

Conventional film-forming material based on acrylic-styrene polymer, titanium dioxide, calcium carbonate, water and additives was obtained (M). The additives include surfactants, dispersants, rheological modifiers and light stabilizers. The sample $\mathrm{M}$ was obtained as follows: in a pearl mill water, additives and $\mathrm{NH}_{3}$ were added and mixed for 5 min followed by the addition of the calcium carbonate and titanium dioxide and further 20 min mixing. In the last step, the conventional film-forming material is obtained by the addition of the acryl-styrene polymer.

\subsection{Preparation of antimicrobial (AM) film-forming materials}

The Ag-nanoparticles were added in the conventional filmforming material at the end of the manufacturing process of MPAS. The dispersion of AgNP in the antimicrobial filmforming materials was realized by the use of an ultrasonic bath, the stability of the colloid was assured by the presence of the anionic surfactant and dispersant agents (table 1).

\subsection{Shelf stability}

Initial formulations were tested for shelf stability under accelerated aging conditions at $50{ }^{\circ} \mathrm{C}$ for 30 days. Samples

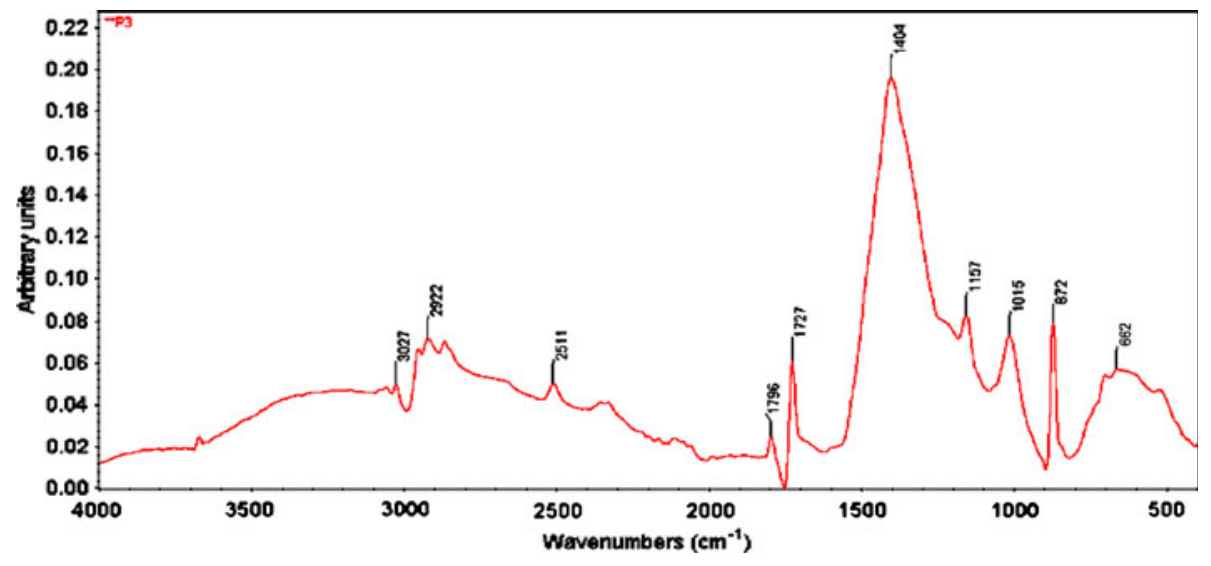

Figure 2. FT-IR spectrum of AM1-AM5 antimicrobial coatings. 
were also evaluated for viscosity change after one month of storage under ambient conditions. All formulations containing AM either had no sedimentation or had sedimentation which could be easily redispersed with low agitation/shaking after the duration of the shelf stability testing. Viscosities were generally stable and are summarized in figure 1.

\section{Results and discussion}

\subsection{IR measurement}

The IR spectra of the synthesized AM1-AM5 antimicrobial coatings present the characteristic peaks of the main
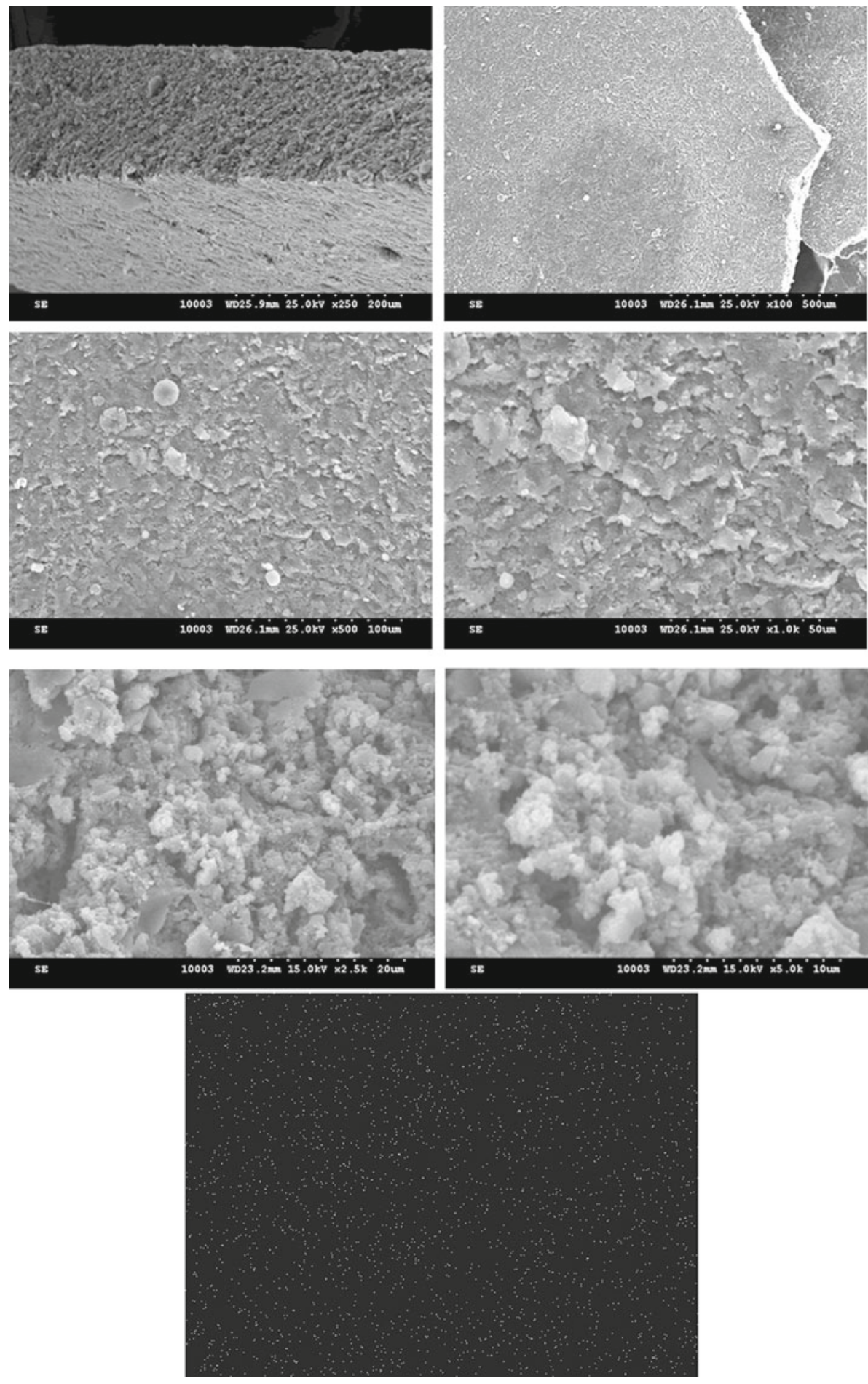

Figure 3. SEM-EDS images of AM1 antimicrobial coating at different magnifications. 
Table 2. Film-forming characteristics.

\begin{tabular}{|c|c|c|c|c|c|c|c|}
\hline Characteristics & Procedure & AM1 & AM2 & AM3 & AM4 & AM5 & MPAS \\
\hline \multicolumn{8}{|l|}{ Liquid product } \\
\hline Appearance & Visual & \multicolumn{6}{|c|}{ homogeneous and viscous } \\
\hline $\begin{array}{l}\text { Brookfield viscosity, rot } 7, \\
\text { speed } 20,23^{\circ} \mathrm{C}, \mathrm{cP}\end{array}$ & SREN ISO 2555:03 & 12000 & 11800 & 11500 & 10500 & 9500 & 8800 \\
\hline Density $\left(\mathrm{g} / \mathrm{cm}^{3}\right)$ & SR ISO $2811 / 02$ & 1.41 & 1.42 & 1.42 & 1.43 & 1.43 & 1.41 \\
\hline $\mathrm{pH}$ & STAS 8619/3-1990 & \multicolumn{6}{|c|}{9} \\
\hline \multicolumn{8}{|l|}{ Dry film } \\
\hline Appearance & Visual & \multicolumn{6}{|c|}{ White plain matter } \\
\hline Washable class & SREN 13300/02 & \multicolumn{6}{|c|}{ Class 1} \\
\hline Coverage (polyester foil) & SREN 6504-3/03, method A & \multicolumn{6}{|c|}{1} \\
\hline $\begin{array}{l}\text { Drying time, } 23{ }^{\circ} \mathrm{C} \text {, film } \\
\text { on glass with applicator } 120 \mu \mathrm{m}(\mathrm{h})\end{array}$ & SREN 29117:98 & \multicolumn{6}{|c|}{1} \\
\hline Resistance to disinfectants (24 h) & SREN ISO 2812-1-2007, method 3 & \multicolumn{6}{|c|}{ No changes on film } \\
\hline
\end{tabular}

components: titanium dioxide (rutile), $\mathrm{CaCO}_{3}$ as well as the absorption bands of ethyl and methyl groups of the acrylic-styrene polymer. Therefore, the spectral bands from 640 to $520 \mathrm{~cm}^{-1}$ are assigned to stretching and deformation vibrations characteristic for Ti-O-Ti bond in the form of rutile. Sharp spectral bands at 1795, 876 and 712 are characteristic to calcium carbonate. The spectral bands at 2926 and $2869 \mathrm{~cm}^{-1}$ are characteristic to methyl and ethyl groups from acrylic-styrene polymer structure. The wide band at $3327 \mathrm{~cm}^{-1}$ is characteristic to associated $\mathrm{OH}$ groups. Regardless of the samples, the IR spectra (peak positions, as well as relative intensity of the peaks) are mainly the same, the spectrum of the AM5 being presented in figure 2.

\subsection{Scanning electron microscopy (SEM)}

A complete characterization of the coatings must include microscopic images. The images were recorded for all the samples on surface as well as in sectional view. In order to be analysed by SEM, the film-forming materials (figure 3) were applied on glass, dried and finally the obtained antimicrobial coatings were detached. At low magnification, a defect free surface is visualized. At higher magnification, SEM images of the antimicrobial coatings reveal typical particles reinforced with polymer matrix composite materials. At $5000 \times$, the polymer matrix as well as the constituent particles and agglomerates of calcium carbonate, hydrated magnesium silicate and titanium dioxide are visualized. At all the magnifications, the samples exhibit a homogenous distribution of the particles and agglomerations. The presence of AgNPs and their homogenous distribution are easily pointed out by elemental EDS map recorded at 500×.

\subsection{Film-forming characteristics}

The film-forming materials as well as the dried coatings were characterized in agreement with the running standards (table 2).

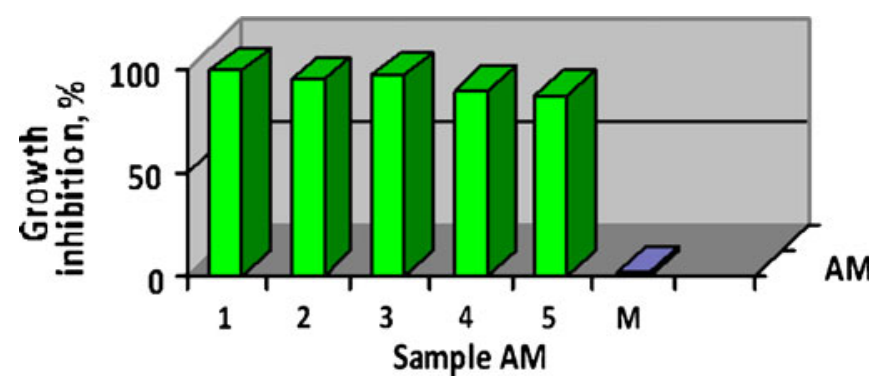

Figure 4. Growth inhibition of AM1-AM5 antimicrobial coatings against Staphylococcus aureus.

\subsection{Antimicrobial tests}

AgNP attaches to the cell membrane and penetrates inside the bacteria. The penetration through the membrane is favoured by the low dimension of the AgNPs; the smaller AgNPs (higher surface area) is the most aggressive for the cell membrane. They attack, preferably, the respiratory chain, leading ultimately to cell's death (Pinto 2010).

The resistance of the antimicrobial coatings to mass infection with the microorganisms was determined according to STAS 12719. This method is basically the direct contact between the AM antimicrobial coatings and the suspension of microorganisms. The AM1-AM5 film-forming materials are applied on filter paper rings, dried and inserted into the cultured dishes with microorganisms. The antimicrobial activity of the AM samples was tested on the following strains of microorganisms: Staphylococcus aureus and Bacillus cereus. The tests were conducted in parallel with a blank sample (a film-forming material without AgNPs) marked M and AM samples. The results are shown in figures 4 and 5 .

As shown in figure 4, all the coatings (AM1-AM5) have antimicrobial activity, with an inhibition capacity between 87 and $99 \%$ in the case of Staphylococcus aureus while the blank sample marked $\mathrm{M}$ does not have antimicrobial activity. The AgNP content positively influences the antimicrobial 


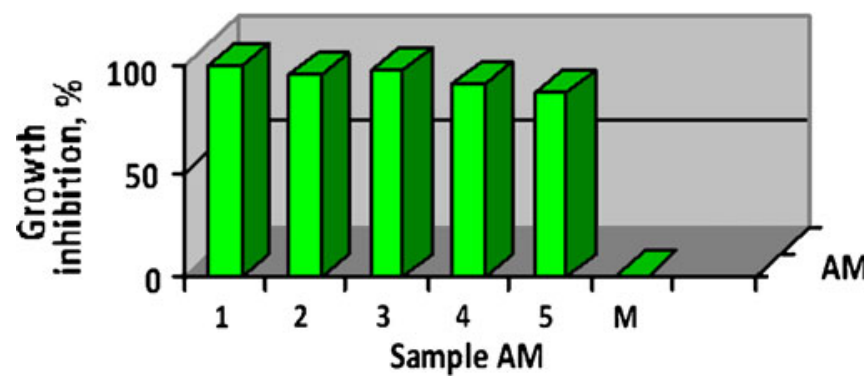

Figure 5. Growth inhibition of AM1-AM5 antimicrobial coatings against Bacillus cereus.

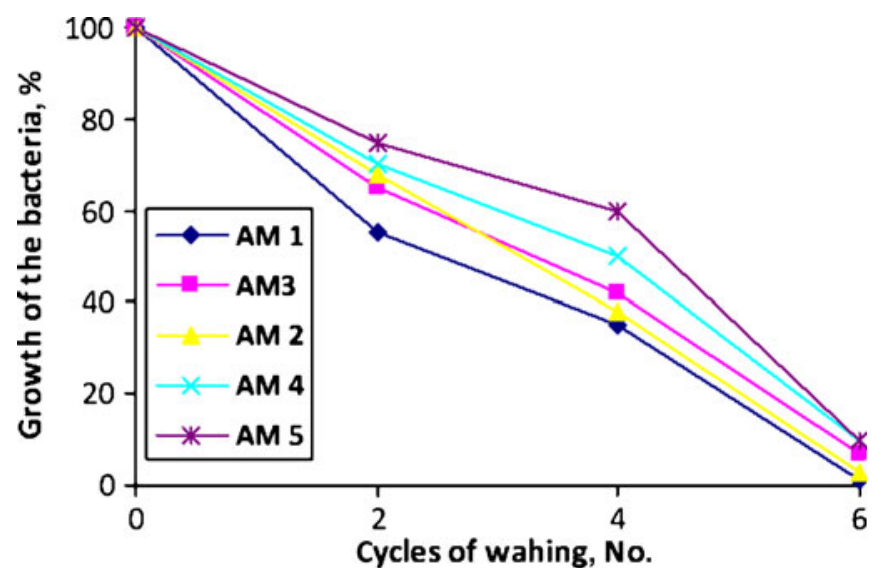

Figure 6. Resistance to Staphylococcus aureus after several cycles of washing (1-6).

activity; the best results were obtained by the sample AM1, in which the AgNP content is $650 \mathrm{ppm}$. Similar results were obtained in the case of Bacillus cereus AM as shown in figure 5 .

The coating materials used in hospitals or for domestic applications are subjected to repeated washing with water and detergent. Due to the repeated washing of the coatings, the antimicrobial activity of the AM1-AM5 against Staphylococcus aureus was analysed after prolonged washing with water and detergent (1-6 cycles $\times 4 \mathrm{~h}$ each cycle). The results are shown in figure 6 . We suppose that bacterial growth inhibition is caused by the presence of large amounts of silver ions (in the presence of increased moisture, the Agnanoparticles release silver ions in excess, which is responsible for its antimicrobial coatings).

It is worth to mention that scrubbing the coating does not adversely affect its effectiveness at preventing the growth of the bacteria. In fact, the data (figure 6) suggest that scrubbing improves the performance, especially at six cycles of washing. Repeated washing and wiping of the paint with a cloth soaked in water or household cleaners containing anionic surfactants does not affect the antibacterial performance of the paint. This is important to maintain the antimicrobial

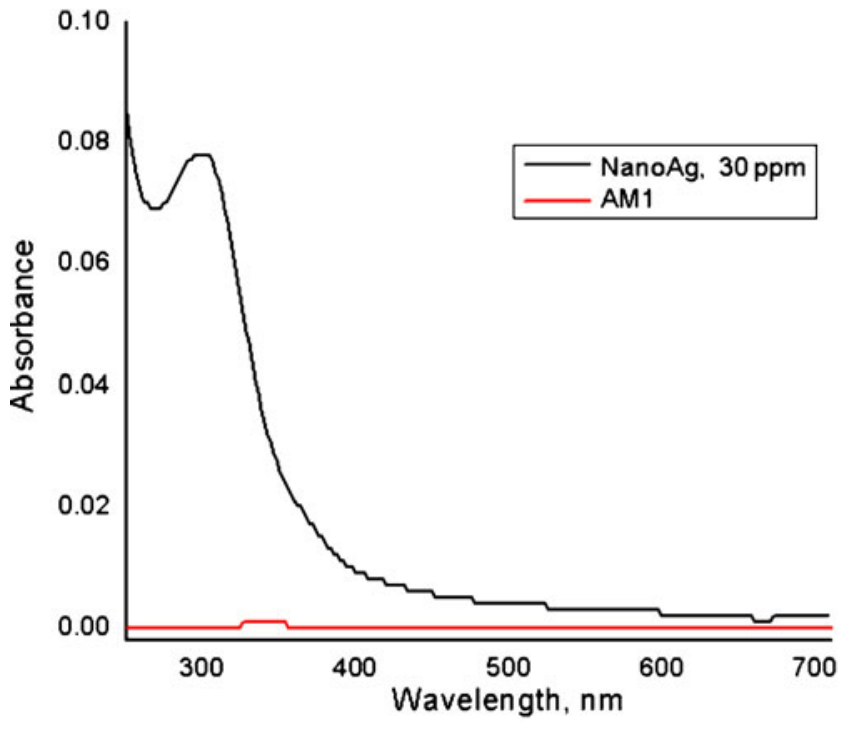

Figure 7. UV-vis spectrum of colloidal silver (nano-Ag, $30 \mathrm{ppm}$ ) and spectrum of solution resulted after washing of sample AM1.

performance of the dry coatings before and after cleaning in hygienic sensitive areas such as hospitals and kitchens.

\subsection{Film stability/nano-Ag release}

In order to test the stability of the films, the coatings were exposed to 6 cycles of washing with water and launder (6 cycles $\times 4 \mathrm{~h}$ each cycle). The resulting solutions were analysed by UV-vis spectroscopy in order to point out that no nano-Ag leaching occurs. UV-vis spectra were recorded for all the samples (AM1-5; only the spectra of the solution resulting for AM1 is presented) using as reference, the solution obtained after the washing of the conventional film. For comparison, a $30 \mathrm{ppm}$ nano- $\mathrm{Ag}$ solution was recorded vs distilled water. It is observed that the washing waters of AM15 samples do not exhibit the characteristic peak of nano- $\mathrm{Ag}$ centred at $300 \mathrm{~nm}$ which proves that no nano-Ag leaching occurs (figure 7).

\section{Conclusions}

A water organic-inorganic dispersion of coating material has been prepared in the system for water soluble acrylic-styrene copolymer-nanoAg (antimicrobial additives).

It was shown that our formulations of film-forming materials containing AgNP in their composition have antimicrobial activity against Staphylococcus aureus and Bacillus cereus. Perhaps, more important is that AgNP-based coating materials that we have formulated have preserved the antimicrobial properties even if they have been subjected to several repeated washings with detergent and water. Based on the UV-vis spectroscopy, no nano-Ag leaching was detected even after prolonged washing. 


\section{References}

Barmatov E B, Medvedev A V and Barmatova M V 2008 Inventors; Particulate silver biocides and methods for biocide use in fracturing fluids. United States Patent 11/942839

Borkow G 2005 Hygienic Coatings \& Surfaces, PRA Coatings Technology Centre, Paris, p. 20

Hung H S and Hsu S H 2007 Nanotechnology 189

Niegisch N, Akarsu M, Csögör Z, Ehses M and Schmidt H 2002 Hygienic coatings (Belgium: Brussels) p. 20
Pica A, Ficai D and Guran C 2012 Rev. Chim. Bucharest 63 459

Pinto V V, Ferreira M J, Silva R, Santos H A, Silva F, Pereira C M 2010 Coll. Surf. A: Physicochem. Eng. Aspects 36419

Ross J 2004 Polym. Paint Color J. 19418

Sauvet G, Dupond S, Kazmierski K and Chojnowski J 2003 J. Appl. Polym. Sci. 751005

Thölmann D, Kossmann B and Sosna F 2003 J. Eur. Coat. 116

Yamauchi G, Riko Y, Yasuno Y, Shimizu T and Funakoshi N 2005 (Manchester, UK: The Paint Research Association) p. 20 